\title{
The Chinese Image on Twitter: An Empirical Study Based on Text Mining*
}

\author{
Ming Xiao \\ Communication University of China, Beijing, China \\ Hongfa Yi \\ Fudan University, Shanghai, China
}

\begin{abstract}
The study use crawler to get 842,917 hot tweets written in English with keyword Chinese or China. Topic modeling and sentiment analysis are used to explore the tweets. Thirty topics are extracted. Overall, $33 \%$ of the tweets relate to politics, and $20 \%$ relate to economy, $21 \%$ relate to culture, and $26 \%$ relate to society. Regarding the polarity, $55 \%$ of the tweets are positive, $31 \%$ are negative and the other $14 \%$ are neutral. There are only $25.3 \%$ of the tweets with obvious sentiment, most of them are joy.
\end{abstract}

Keywords: Chinese image, topic modeling, sentiment analysis, text mining, Twitter

\section{Introduction}

National image is the reflection of the comprehensive national strength (scilicet the sum of the hard power and the soft power), and the overall appearance which a country shows in the international community, as well as the international feelings and evaluation of the country's comprehensive national strength, the core values and the overall appearance. National image has the following characteristics: (1) the image of the country is a reflection of overall strength and core values; (2) it is mainly reflected in media; and (3) it is also a national public image.

In the past, these country representations, or images, were investigated through the use of survey research techniques. One drawback of survey research techniques is that the respondent taking a survey is usually aware that he/she is being measured, and this very fact might result in more calculated and less candid responses.

The advent and rapid growth of social media platforms offers a new context in which to study how countries are represented in the minds of media users. The social media context offers an environment where individuals express their thoughts without the encumbrance of a formal measurement session.

Twitter, a widely used channel for online communication, is a micro blogging website that allows users a limit of 140 characters per post, or "tweet". Twitter allows users to engage in public discourses on certain issues through its website and its app for mobile devices. Based on a report published by Twitter (2015), about 316 million active unique users throughout the world send more than 500 million tweets per day. The service has facilitated online users in their participation in collective action, dissemination of emerging news, and exchange of opinions (Runge et al., 2013).

\footnotetext{
" Acknowledgments: This work is supported by the Asia Media Research Center, Communication University of China. Ming Xiao, Associate Professor, Journalism Department, Communication University of China. Hongfa Yi, Doctor-postgraduate, Journalism Department, Fudan University.
} 
Studies have shown that, the sentiment of Twitter users and the result of traditional survey and voting share a high degree of consistency, in aspects of consumer confidence and political electoral issues, the correlation between them is up to $80 \%$ (O'Connor, Balasubramanyan, Routledge, \& Smith, 2010).

In the present study, we use text mining techniques to analyze HOT TWEETs in English which involves China or Chinese issues. We conduct present study in order to comprehend and describe topics and attitude which Twitter users concerned and showed when they mentioned China, and to reveal the difference between tweets and attitude from media account and from public account.

\section{Methodology}

There are more than 30 kinds of languages in Twitter platform, but the main language is English. The research scope of present study is the English version, and the subject is hot tweets related to China which are released by overseas media and public. We adopt the method of text mining to dig into and process unstructured text of Tweets. The procedure includes four steps which are text acquisition, segmentation and filtering, topic modeling, and sentiment analysis.

\section{Data Collection}

In the process of text acquisition, we searched through hot tweets using key words "China" or "Chinese" (letters are not case-sensitive). Hot tweets are the tweets that retweeted or favorited tweets. The main reason we searched through hot tweets but not all tweets is that we considered the influence of hot tweets and to reduce workload. We searched in English because the English is mainly used among Twitter users, and most tweets are released in English. Twitter has begun to operate in March 2006, we started to collect data in early 2014, so time period of the research is from March 2006 to December 2013.

The crawler software Gooseeker was used to crawled the hot tweets which had searched out. GooSeeker is a Firefox browser plugin, consists of two parts: MetaStudio that definite extraction rules and DataScraper that is used for collecting information. The original data obtained by the crawler technology included four fields: respectively were "user name", "nickname", "tweet release date" and "text". The "user name" and "nickname" is the data of user attribute; "Tweet release date" is the data of tweets attribute; "Tweet Text" is the data of content which is in form of text as well as unstructured data, which is the exact subject of our study.

Since this study is concerned with the image of China presented by foreigners, we removed the tweets published by Chinese people or the Chinese institutions. First of all, we decided that "nickname" of users contained Chinese characters are Chinese, deleted their tweets, the amount of 26,318. Second, we identified that if the user name or "nickname" contains key words "China" or "Chinese", these users are also associated with China, removed their tweets, the amount of 53,212. The ultimate effective hot tweets we got are in a total of 842,917 .

\section{Data Preprocessing}

At the step of pretreatment, we mainly adopted natural language processing (NLP) technique to segment and filter the text of tweets.

We conducted the research of China-related tweets on Twitter in English, using English word segmentation which is based on Spaces and punctuation marks.

The point of performing the process of filtering was to remove html links in text, @ names, punctuation, and unnecessary spaces. The process also filtered out the non-English letters, numbers, words less than or equal to 3 characters (such as "the", "or", "and", and the vast majority of meaningless function words), at the same 
time filtered out the Stop Words on the basis of the Stop Words list. We employed the Python nltk packet to segment and filter the text of pushed tweets, and operated it with natural language processing (NLT) technique.

Finally, all the English letters converted to lower-case.

\section{Topic Modeling}

Topic modeling is the most crucial step in this study. Our research built LDA (latent Dirichlet allocation) model on topics of China-related tweets using Stanford TMT 0.4.0. Stanford TMT 0.4.0 was developed by The Stanford Natural Language Processing Group, it is based on JAVA, written by Scala and possesses complete API document. As a approach based on unsupervised machine learning technology, LDA modeling does not identify tweets subjectively, and is able to find latent theme for the text more objectively.

After testing repeatedly, we chose the results that with maximum iterations -1000 and with the number of common words filtering - 20, finally extracted 30 topics from more than 800 thousands tweets.

The results of extracted 30 topics have shown top 20 keywords of each theme and the contribution degree of each keyword.

\section{Sentiment Analysis}

In current research we identified the polarity and emotion using sentiment package of $\mathrm{R}$ language with application of machine learning techniques and Naive Bayes algorithm.

Sentiment is a kind of $\mathrm{R}$ package for emotional analysis which classifies different types of emotion (joy, anger, sadness, surprise, fear, and disgust) and identifies different polarities like negativity, positivity and neutrality in a text with employment of naive bayesian classifiers. Introduced as follow:

Sentiment package of $\mathrm{R}$ language grades for every possible polarity of each tweets while it identifies them. There are two possible grades for each tweet: negative and positive. When the difference between two polarity score was big, the polarity fitting would be categorized in polarity that grades higher, and when the difference was small, the polarity fitting would be categorized in neutrality.

The sentiment package of R language could classify six types of emotion: joy, anger, sadness, surprise, fear, and disgust. While analyzing the types of emotion, it would grade for every possible type. If there were particular prominent score among six types, the actual emotion of the tweet would be fitting for that category; if the difference between scores of six types is unnoted, the emotion would be fitting for "unknown". So we can see, if a tweet was fitting for a certain type of emotion, this emotion must be very strong.

\section{Result}

\section{The Distribution of the Amount of China-related Tweets}

After data capturing, we received a total of 842,917 valid Popular Tweets article. From the prospective of year distribution, there is continuous increase of Popular China-related tweets from 2006 to 2012. However the amount of China-related hot tweets has decreased in 2013 relative to 2012.

\section{Topic Distribution}

In the present study we condensed 800 thousands China-related hot tweets into 30 topics with LDA modeling. The name of each topic and its percentage of all subjects are shown in Table 1 to Table 4.

As you can see, there are three topics among China-related hot tweets that are related to diet, and they could be divided according to different scenarios, respectively: "Diet and family life", "Food and entertainment", and "Diet and school life", accounted for $10.98 \%$ of the total. 


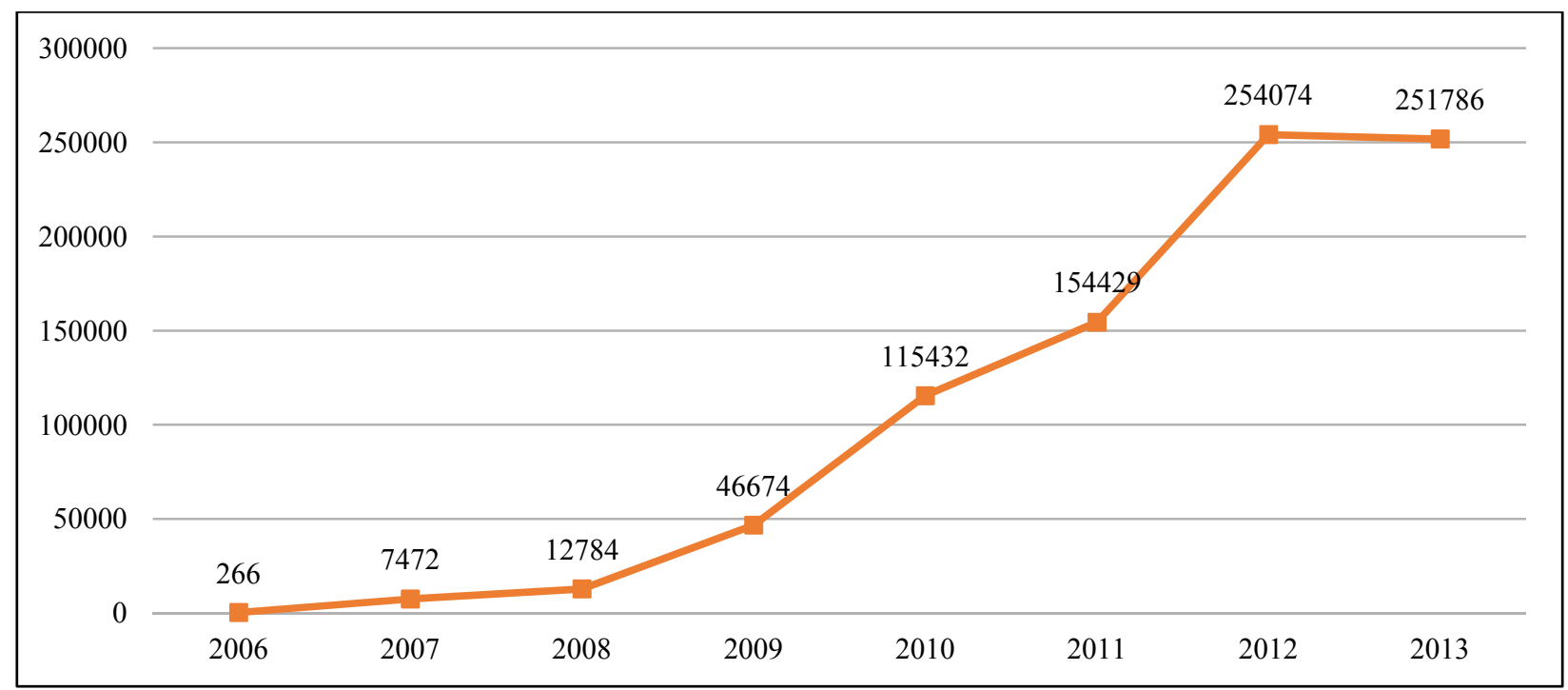

Figure 1. The change of the number of China-related hot tweets in every year $(N=842,917)$.

After the theme of "diet", top five topics that are accounted most of the total is, in order, "The situation in Asia", "Presidential Election of United States", "Financial market", "Social media", "Economic growth", their cumulative percentage is $26 \%$, which is more than a quarter.

In the studies of National Image, there is common classification of topics: those are Politic, Economy, Society, and Culture, which is also the way of classification used in traditional method of survey and content analysis.

According to the above mentioned approach of classification, nine topics discovered with LDA modeling in the study, "The situation in Asia", "Presidential Election of United States", "Regional conflict", "Tibet issue", "Media censorship", "Human rights issue", "Media review", "The issue of HK and Taiwan", "Political scandal", and "Family planning" belong to the category of Politic, the proportion is $32.6 \%$.

Table 1

The Topics Belong to Politic

\begin{tabular}{lll}
\hline Category & Topic & Proportion \\
\hline & The situation in Asia & $5.75 \%$ \\
& Presidential Election of United States & $5.66 \%$ \\
Regional conflict & $4.16 \%$ \\
Tibet issue & $4.07 \%$ \\
Politic & Human rights issue & $3.01 \%$ \\
& Media review & $3.15 \%$ \\
& The issue of Hongkong and Taiwan & $2.90 \%$ \\
& Political scandal & $2.05 \%$ \\
& Family planning & $1.84 \%$ \\
& Total & $32.6 \%$ \\
\hline
\end{tabular}

Topics that belong to the category of Economic are "Financial market", "Economic growth", "Luxury market", "Apple products", "Pollution and health", "Aerospace and lunar exploration project", the total proportion is $20 \%$. 
Table 2

The Topics Belong to Economic

\begin{tabular}{lll}
\hline Category & Topic & Proportion \\
\hline & Financial market & $5.20 \%$ \\
& Economic growth & $4.68 \%$ \\
Economic & Luxury market & $3.52 \%$ \\
& Apple products & $2.70 \%$ \\
& Pollution and health & $2.30 \%$ \\
& Aerospace and lunar exploration project & $1.55 \%$ \\
& Total & $20.0 \%$ \\
\hline
\end{tabular}

Seven topics belong to the category of Culture, those are "Giant panda and The Great wall", "Reading and Proverbs", "The Hollywood films", "School and learning", "Language learning", "Korean pop culture", and "Traditional medicine", the proportion is $21 \%$.

Table 3

The Topics Belong to Culture

\begin{tabular}{lll}
\hline Category & Topic & Proportion \\
\hline & Giant panda and The Great wall & $3.47 \%$ \\
& Reading and Proverbs & $3.15 \%$ \\
The Hollywood films & $3.11 \%$ \\
Culture & School and learning & $3.10 \%$ \\
& Language learning & $2.90 \%$ \\
& Korean pop culture & $2.70 \%$ \\
& Traditional medicine & $2.60 \%$ \\
\hline
\end{tabular}

Eight themes belong to the category of Society, those are "Social media", "Diet and family life", "Natural disasters and public security", "Food and entertainment", "Diet and school life", "The Olympic", "City life" and "Basketball and football", the proportion is $26.4 \%$.

Table 4

The Topics Belong to Society

\begin{tabular}{lll}
\hline Category & Topic & Proportion \\
\hline & Social media & $4.74 \%$ \\
& Diet and family life & $4.46 \%$ \\
& Natural disasters and public security & $3.63 \%$ \\
Society & Food and entertainment & $3.56 \%$ \\
& Diet and school life & $2.95 \%$ \\
& The Olympic & $2.58 \%$ \\
& City life & $2.35 \%$ \\
& Basketball and football & $2.15 \%$ \\
& Total & $26.40 \%$ \\
\hline
\end{tabular}

\section{Polarity Analysis of Different Year and Different Topics}

We found out through polarity analyzing that among 800 thousands China-related tweets in English, there are $55.17 \%$ of tweets that are positive, $31.11 \%$ are negative and $13.72 \%$ are neutral. 
The data in the year of 2006 was excluded because of the small amount. The polarity change from 2007 to 2013 is insignificant. The standard deviation of negative evaluation was $1.87 \%$, the standard deviation of positive evaluation was $1.32 \%$ and the standard deviation of neutral evaluation was less than $1 \%$ which changed in the most minimal scope.

Table 5

The Annual Change of Polarity (2007-2013)

\begin{tabular}{lll}
\hline & Means (data from 2006 was excluded) & Standard deviation \\
\hline Negative & $31.99 \%$ & $1.87 \%$ \\
Neutral & $13.20 \%$ & $0.90 \%$ \\
Positive & $54.81 \%$ & $1.32 \%$ \\
\hline
\end{tabular}

According to the known, means of the positive evaluation of 2008 is the lowest, $52 \%$, but the means of the negative evaluation is the highest, 35.68\%. Major events that occurred in 2008 are the Beijing Olympic, The earthquake in Wenchuan, The milk powder scandal and the global financial crisis.

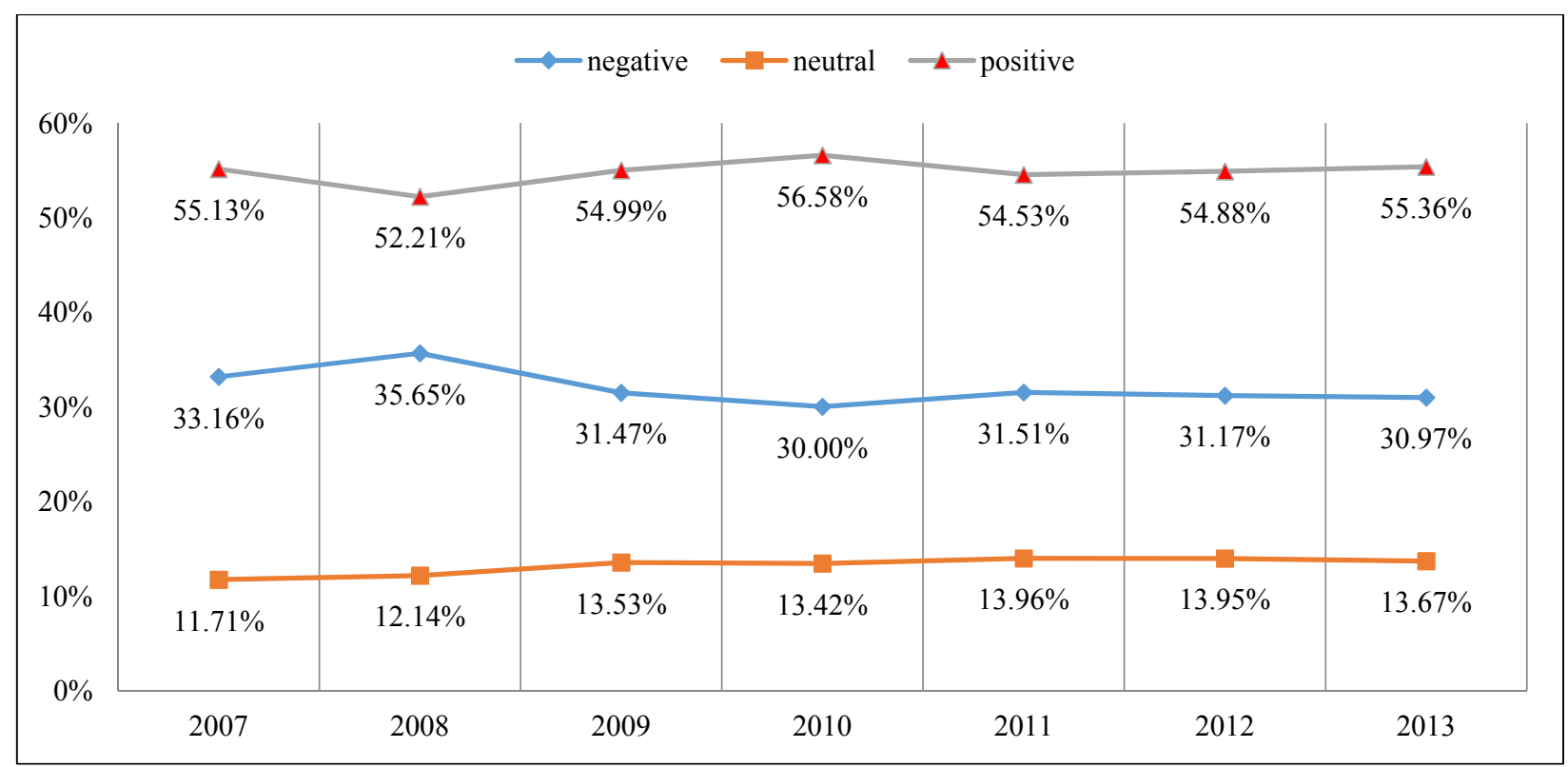

Figure 2. The distribution of polarity from 2007 to 2013.

If we classified topics into Politic, Economy, Culture and Society like in traditional way, the proportion of positive evaluation in political themes is $54.73 \%$ which is slightly lower than the similar evaluation in economic, social and cultural theme.

Table 6

The Polarity of Four Categories Issues

\begin{tabular}{llll}
\hline Category of topics & Negative & Neutral & Positive \\
\hline Politic & $31.55 \%$ & $13.73 \%$ & $54.73 \%$ \\
Economy & $31.55 \%$ & $13.21 \%$ & $55.24 \%$ \\
Culture & $30.08 \%$ & $14.24 \%$ & $55.68 \%$ \\
Society & $30.30 \%$ & $14.24 \%$ & $55.46 \%$ \\
\hline
\end{tabular}




\section{Sentiment Analysis of Different Year and Different Topics}

Overall, only $25.54 \%$ of researched tweets showed strong emotion. Nearly three-quarters of the tweets did not show strong emotions, emotional analysis fit for the "unknown".

Diachronically, China-related tweets have shown stronger and stronger emotion with the passage of time. Only $20.1 \%$ of tweets have shown intense emotion in 2007, but in 2013 the percentage has been raised to 26.5\%. This suggests that Twitter users are increasingly inclined to express strong emotions in terms of Chinese issue.

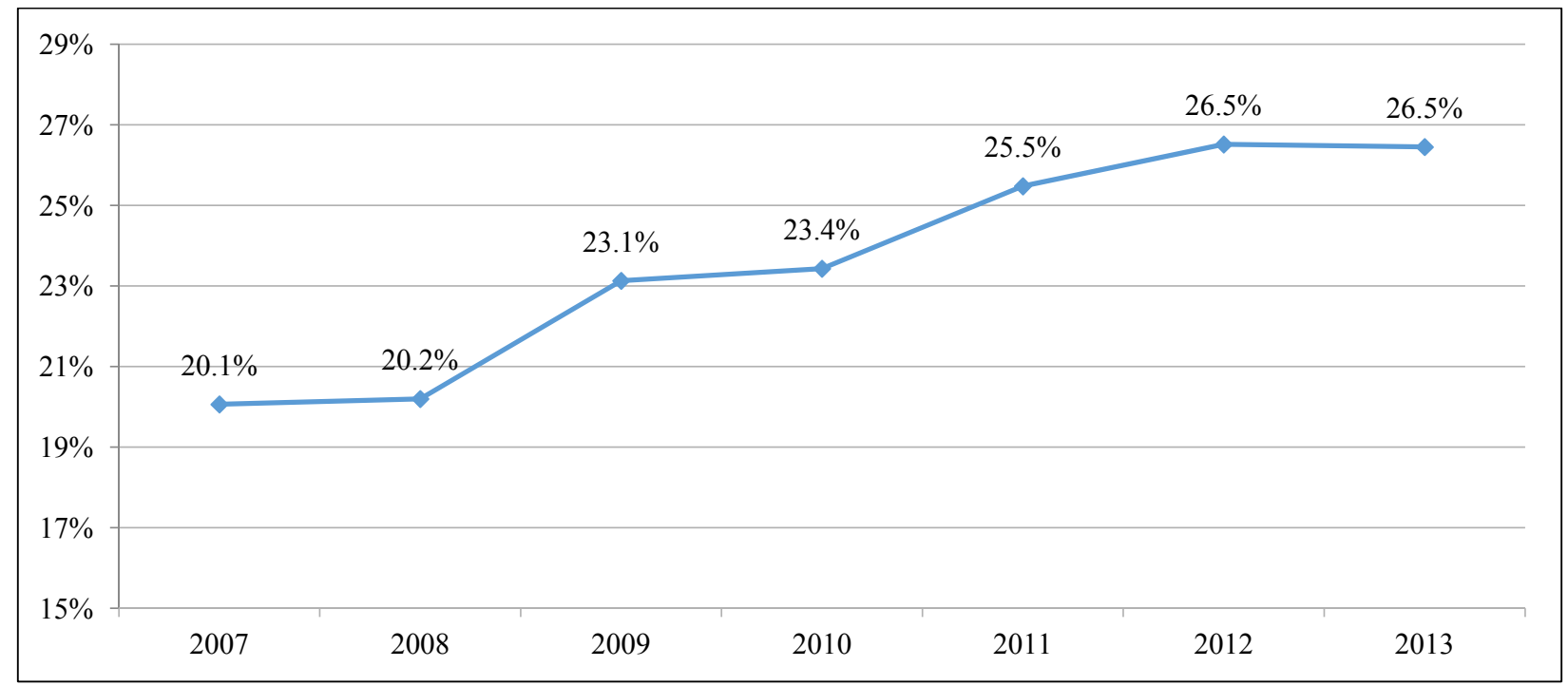

Figure 3. The distribution of showed strong emotion from 2007 to 2013.

In general, the highest proportion of expressed emotion was joy, accounting for $14.19 \%$; followed by anger, accounting for $3.49 \%$; in third place was sadness, the ratio was $3.07 \%$.

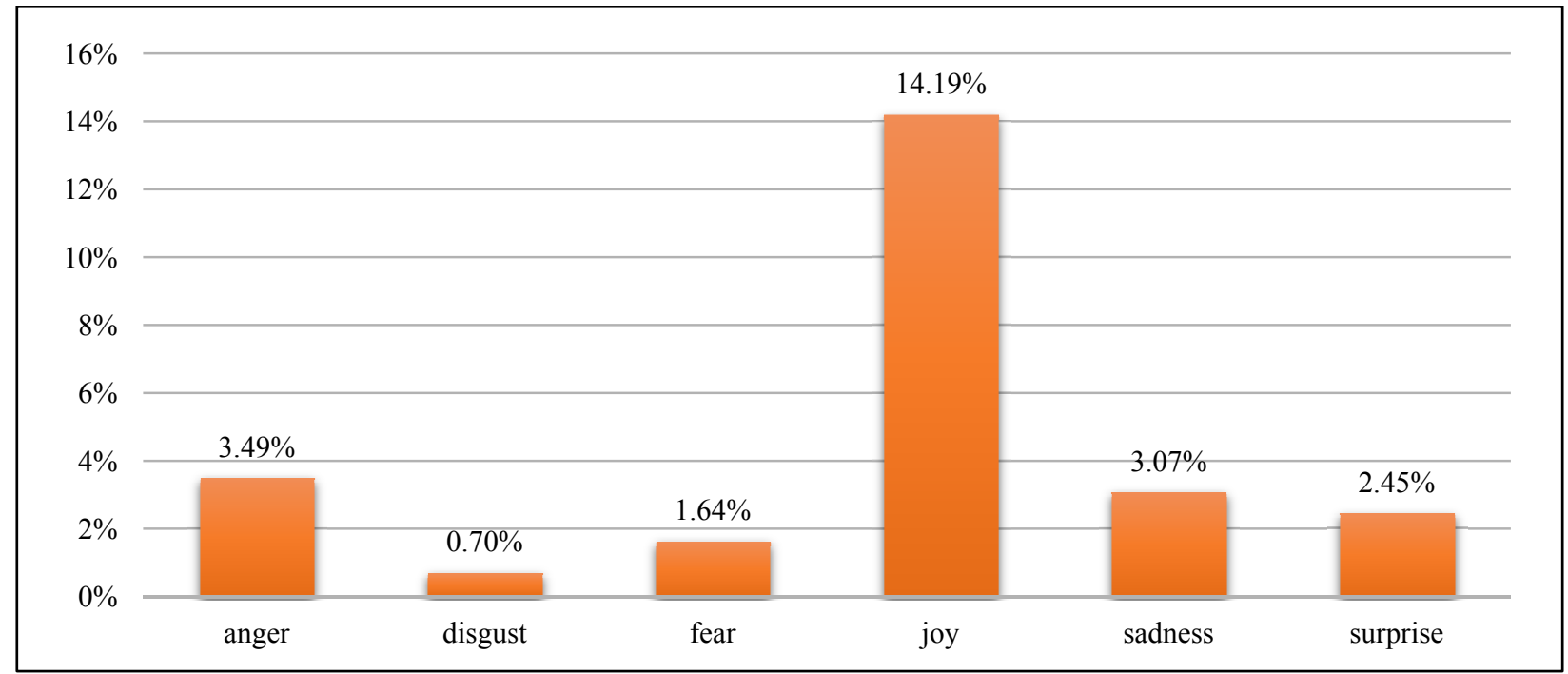

Figure 4. The distribution of showed strong emotion.

The standard deviation of "joy" is the greatest according to emotional change in years, which shows that the changing range of this emotion is maximum. 
Table 7

The Annual Variability of Emotion

\begin{tabular}{lll}
\hline & Means (data from 2006 was excluded) & Standard deviation \\
\hline Anger & $3.33 \%$ & $0.34 \%$ \\
Disgust & $0.66 \%$ & $0.14 \%$ \\
Fear & $1.59 \%$ & $0.16 \%$ \\
Joy & $12.81 \%$ & $1.90 \%$ \\
Sadness & $2.90 \%$ & $0.29 \%$ \\
Surprise & $2.33 \%$ & $0.27 \%$ \\
\hline
\end{tabular}

"Joy" has the largest standard deviation, 1.9\%, which means that the changing range of this emotion is maximum; "anger" has shown the second large changing range, whose standard deviation is $0.34 \%$. The other four classifications of emotion have changed slightly.

The change of "Joy" has presented the increasing trend, suggesting that there are more and more China-related tweets with joyful emotion.

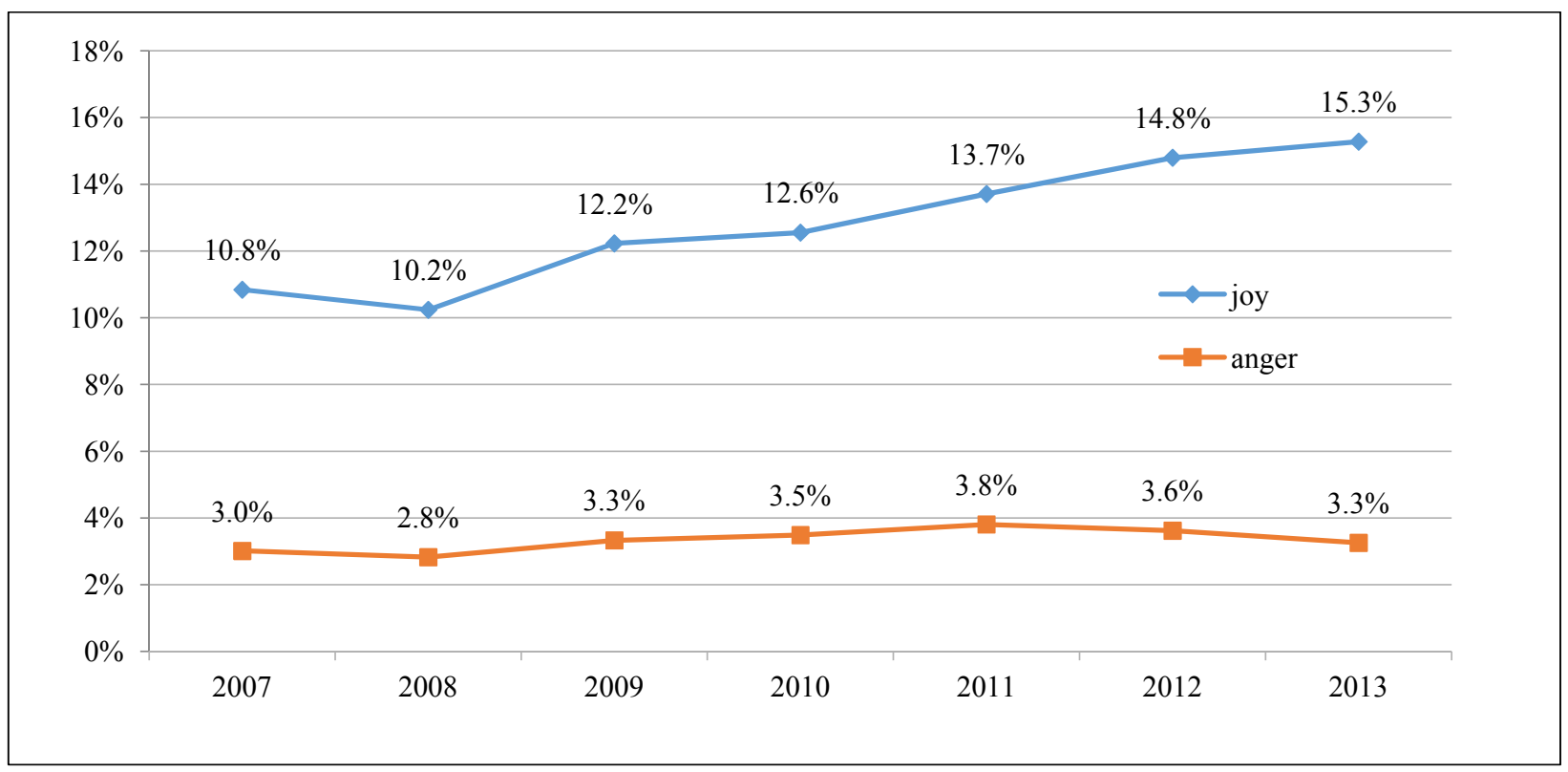

Figure 5. The proportion of change of "Joy" and "Anger".

The proportion of intense feeling for social issues is the largest among four classifications of traditional method, 27\%; however, the proportions of strong emotion have been showed for economic, political issues are less than $25 \%$.

Table 8

The Emotion of Four Categories Issues

\begin{tabular}{llllllll}
\hline & Joy & Anger & Sadness & Surprise & Fear & Disgust & Strong emotion(total) \\
\hline Politic & $13.61 \%$ & $3.56 \%$ & $3.00 \%$ & $2.38 \%$ & $1.63 \%$ & $0.69 \%$ & $24.87 \%$ \\
Economy & $13.21 \%$ & $3.40 \%$ & $2.98 \%$ & $2.47 \%$ & $1.59 \%$ & $0.69 \%$ & $24.34 \%$ \\
Culture & $15.22 \%$ & $3.46 \%$ & $3.19 \%$ & $2.51 \%$ & $1.66 \%$ & $0.71 \%$ & $26.74 \%$ \\
Society & $15.21 \%$ & $3.58 \%$ & $3.25 \%$ & $2.56 \%$ & $1.69 \%$ & $0.72 \%$ & $27.01 \%$ \\
\hline
\end{tabular}




\section{Discussion}

In this section we will compare the account of mass media and public account. There are accounts of mass media as well as public accounts in Twitter.

We constructed a media account list based on the organization accounts that were authorized by Twitter which included mass media account as much as possible and got a total of 1,512 accounts. Among all China-related tweets in English that we have analyzed there are 190,052 tweets pushed by 1,512 media accounts whose proportion is $23 \%$, and there are 652,865 tweets pushed by public accounts whose proportion is $77 \%$.

The number of tweets released by media accounts has increased, in 2006 there were 266 tweets about China, but in 2013 have been raised to 61821 tweets. China-related tweets pushed by public accounts have begun to appear in 2007, the amount of tweets has rapidly grown from 2007 to 2012, and in 2012 the amount has reached its peak: more than 200 thousands, but in 2013 it started to decrease, which might have been affected by the decline of Twitter.

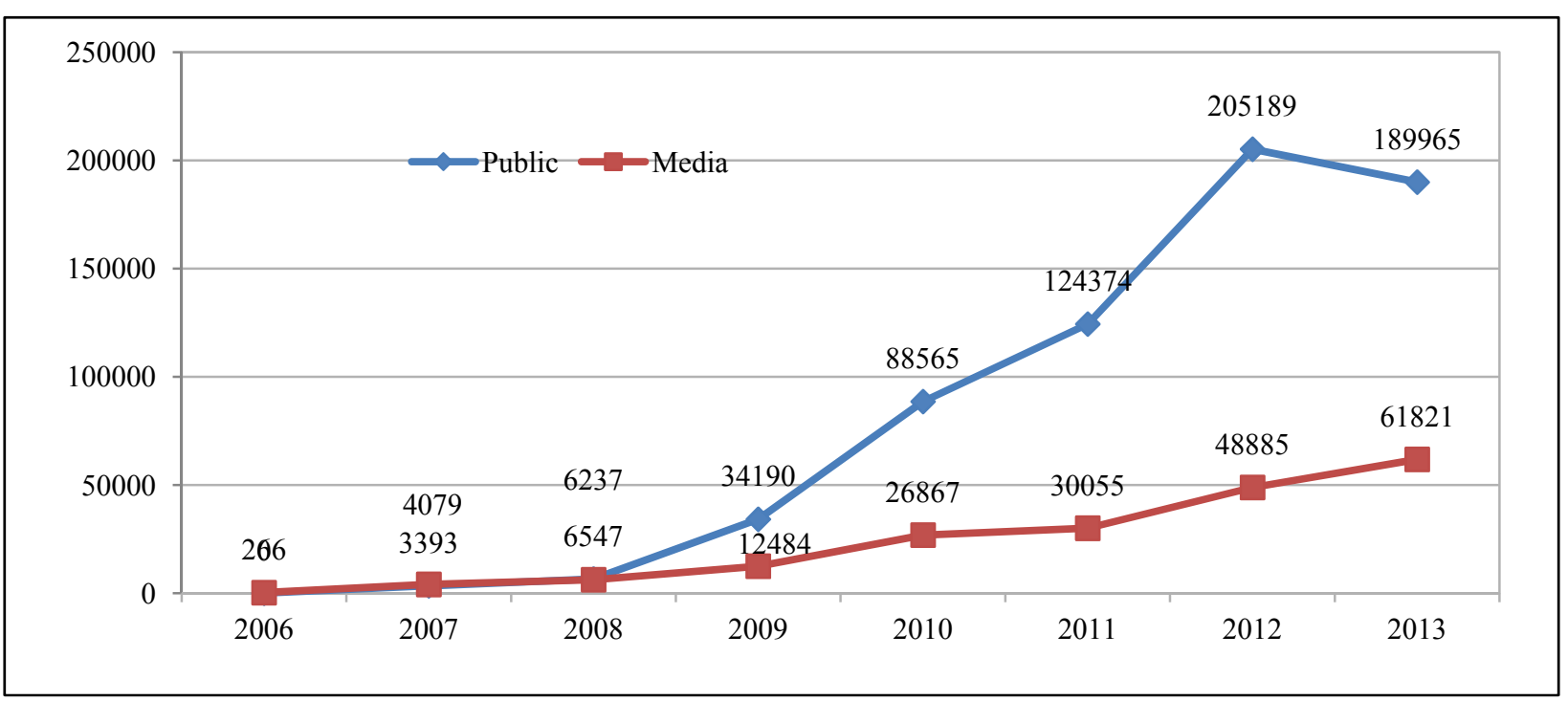

Figure 6. The change of the number from media and public account in every year.

\section{Types of Issues that Media and Public Accounts Concern}

Compared to public accounts, media accounts pay more attention to the macro issues such as political and economic.

Table 9

The Four Category Issues From Media and Public Accounts

\begin{tabular}{llllll}
\hline & Politic & Economy & Culture & Society & Total \\
\hline Public & $30.35 \%$ & $17.72 \%$ & $23.75 \%$ & $28.19 \%$ & $100 \%$ \\
Media & $38.62 \%$ & $27.90 \%$ & $12.74 \%$ & $20.73 \%$ & $100 \%$ \\
\hline
\end{tabular}

\section{Polarity Analysis of Tweets of Media and Public Accounts}

The proportion of negative evaluation of media accounts tweets is $35.7 \%$ which is $6 \%$ higher than the ratio of public accounts tweets' $29.8 \%$. In comparison with media accounts the proportions of positive and neutral evaluation of public tweets are higher. 
The proportion of negative evaluation of Chinese politic from media accounts is the highest, $36.5 \%$, followed by the proportion of economic evaluation, 35.5\%. In comparison with public accounts, media accounts tended to evaluate negatively.

\section{Sentiment Analysis of Tweets Pushed by Media and Public}

In general, $27.84 \%$ of public accounts tweets have shown strong emotion, at the same time $17.67 \%$ of media accounts tweets have shown strong feelings. Public accounts tended to express intense emotion when it is related to China.

$15.8 \%$ of public accounts tweets have shown "joy", while $8.6 \%$ media accounts tweets have shown similar emotion. Public accounts' proportion of the expression of other five kinds of emotion were a bit higher than media accounts'.

Public accounts have higher proportion of strong emotional expression on social theme than the other themes. However media accounts have a bit higher proportion of strong emotional expression on cultural theme.

Public accounts expressed emotion on Politic, Economy, Culture and Society with slight proportional difference. It is the same with media accounts.

Table 10

The Polarity of Four Category Issues From Media and Public Accounts

\begin{tabular}{lllll}
\hline & & Positive & Negative & Neutral \\
\hline Public & Politic & $55.3 \%$ & $30.2 \%$ & $14.6 \%$ \\
& Economy & $56.0 \%$ & $29.9 \%$ & $14.1 \%$ \\
& Culture & $55.8 \%$ & $29.5 \%$ & $14.7 \%$ \\
& Society & $55.7 \%$ & $29.7 \%$ & $14.6 \%$ \\
\multirow{3}{*}{ Media } & Total & $55.7 \%$ & $29.8 \%$ & $14.5 \%$ \\
& Politic & $52.4 \%$ & $36.5 \%$ & $11.1 \%$ \\
& Economy & $53.4 \%$ & $35.5 \%$ & $11.1 \%$ \\
& Culture & $54.7 \%$ & $34.2 \%$ & $11.2 \%$ \\
& Society & $53.9 \%$ & $34.8 \%$ & $11.3 \%$ \\
& Total & $53.3 \%$ & $35.7 \%$ & $11.0 \%$ \\
\hline
\end{tabular}

Table 11

The Emotion of Four Category Issues From Media and Public Accounts

\begin{tabular}{|c|c|c|c|c|c|c|c|c|}
\hline & & Anger & Disgust & Fear & Joy & Sadness & Surprise & Unknown \\
\hline \multirow[t]{5}{*}{ Public } & Politic & $3.6 \%$ & $0.7 \%$ & $1.8 \%$ & $15.3 \%$ & $3.3 \%$ & $2.5 \%$ & $72.7 \%$ \\
\hline & Economy & $3.5 \%$ & $0.8 \%$ & $1.7 \%$ & $15.2 \%$ & $3.3 \%$ & $2.7 \%$ & $72.8 \%$ \\
\hline & Culture & $3.5 \%$ & $0.7 \%$ & $1.7 \%$ & $16.1 \%$ & $3.4 \%$ & $2.6 \%$ & $72.1 \%$ \\
\hline & Society & $3.6 \%$ & $0.7 \%$ & $1.7 \%$ & $16.1 \%$ & $3.4 \%$ & $2.7 \%$ & $71.7 \%$ \\
\hline & Total & $3.6 \%$ & $0.8 \%$ & $1.7 \%$ & $15.8 \%$ & $3.4 \%$ & $2.6 \%$ & $72.2 \%$ \\
\hline \multirow[t]{5}{*}{ Media } & Politic & $3.4 \%$ & $0.5 \%$ & $1.3 \%$ & $8.3 \%$ & $2.1 \%$ & $1.9 \%$ & $82.6 \%$ \\
\hline & Economy & $3.2 \%$ & $0.5 \%$ & $1.3 \%$ & $8.5 \%$ & $2.1 \%$ & $2.0 \%$ & $82.4 \%$ \\
\hline & Culture & $3.3 \%$ & $0.6 \%$ & $1.3 \%$ & $8.8 \%$ & $2.0 \%$ & $2.1 \%$ & $81.8 \%$ \\
\hline & Society & $3.5 \%$ & $0.5 \%$ & $1.3 \%$ & $8.7 \%$ & $2.1 \%$ & $1.9 \%$ & $82.0 \%$ \\
\hline & Total & $3.3 \%$ & $0.5 \%$ & $1.3 \%$ & $8.6 \%$ & $2.1 \%$ & $1.9 \%$ & $82.3 \%$ \\
\hline
\end{tabular}

\section{Conclusion}

The present study focused on the way that how Twitter users present China-related issues using NLP 
technology for data preprocessing and the Crawlers for data capturing, adopting topic modeling and emotional analysis for processing the data, and drew conclusions as follow:

(1) In terms of quantity, the number of China-related HOT tweets in English has been increasing annually, which indicates that international society has been paying more and more attention on China and Chinese issues. Especially popular China-related tweets pushed by media accounts maintained a steady rising trend.

(2) "Diet" has become most popular theme within HOT China-related tweets, more than one tenth of tweets are concerned about China.

(3) The proportion of tweets on political topic within all HOT China-related tweets is the highest among four categories classified in traditional method. In comparison with public accounts media accounts pay more attention on macro issues such as politic and economy.

(4) HOT China-related tweets were positive in general, especially the proportion of negative polarity of public accounts tweets is lower than $30 \%$, and the proportion of similar polarity of media accounts is $35.75 \%$.

(5) Generally, only the quarter of all China-related HOT tweets have shown significant emotion, public accounts have expressed stronger emotion in comparison with media accounts.

(6) The result of data processing suggests that the expression of "joy" took first place, followed by "Anger" and "sadness".

\section{References}

Blei, D., Ng, A., \& Jordan, M. (2003). Latent Dirichlet allocation. Journal of Machine Learning Research, 3, 993-1022.

Hofmann, T. (2001). Unsupervised learning by probabilistic latent semantic analysis. Machine Learning, 42(1), 177-196.

O'Connor, B., Balasubramanyan, R., Routledge, B. R., \& Smith, N. A. (2010). From Tweets to Polls: Linking text sentiment to public opinion time series (p. 559). Tepper School of Business.

Peijing, Z., \& Lei, S. (2012). A review of the research on the topic modeling method of micro-blog text based on LDA. Library and Information Service, 12, 120-126.

Yanyan, Z. (2010). Text sentiment analysis. Journal of Software, 8, 1835. 